\title{
¿Cuál es la mejor cadena de suministro para frutas perecederas, lean 0 ágil?
}

\section{Which is the best supply chain for perishable fruits, Lean or Agile?}

JAVIER ARTURO ORJUELA-CASTRO ${ }^{1,2}$

FREDY SANTIAGO MORALES-AGUILAR ${ }^{1}$

LAURA FERNANDA MEJÍA-FLÓREZ'

Frutas perecederas en un puesto de venta de la plaza Las Ferias en Bogotá.

Foto: J.A. Orjuela-Castro

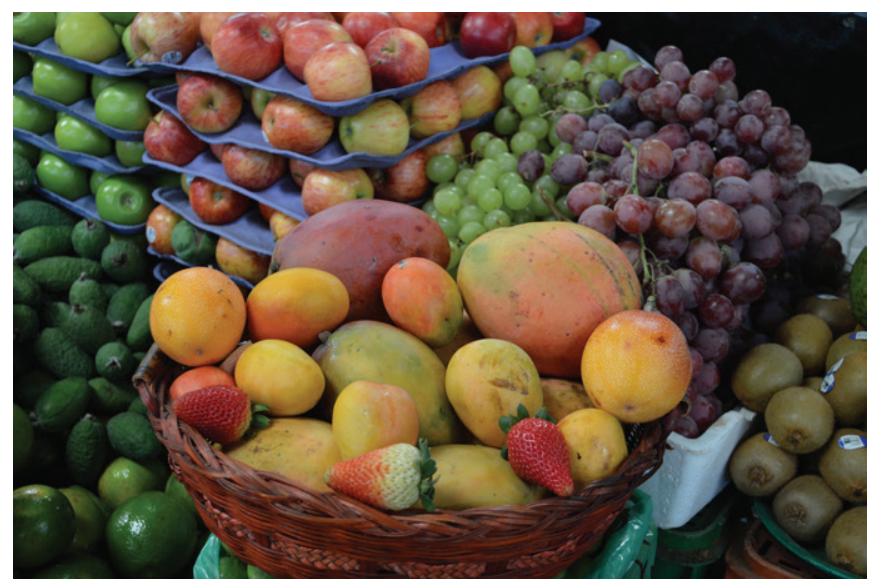

\section{RESUMEN}

Se estima que un tercio de la producción mundial de frutas se pierde o se daña, en los países en desarrollo las pérdidas poscosecha a menudo son superiores al $50 \%$, el crecimiento del consumo mundial genera diversos problemas de abasto y distribución a lo largo de la cadena de suministro. Este artículo evalúa la estructura de la cadena del mango en Cundinamarca-Bogotá, mediante un modelo de dinámica de sistemas, la estructura actual se evaluó frente a las cadenas lean y ágil. Las estructuras se estudiaron respecto a las medidas de desempeño de calidad, capacidad de respuesta, operaciones logísticas y costos. Se encuentra que las cadenas lean y ágil mejoran el desempeño de la cadena en todos los casos, aunque no para todos los actores, se evidencia la existencia de trade-off, lo que presenta la necesidad de evaluar a futuro estructuras combinadas ágil y lean para la cadena de suministro de frutas perecederas.

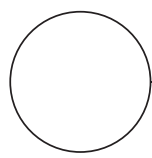

Palabras clave adicionales: cadena de suministro de frutas; estructura; aplicaciones de dinámica de sistemas; perecedero; mango; Cundinamarca-Colombia.

\section{ABSTRACT}

It is estimated that one-third of global fruit production is lost or damaged. In developing countries, post-harvest losses that often exceed $50 \%$, together with the growth of global consumption, generate various problems of supply and distribution throughout the supply chain. This article evaluates the structure of the mango

\footnotetext{
1 Facultad de Ingeniería, Ingeniería Industrial, Grupo GICALyT, Universidad Distrital Francisco José de Caldas, Bogotá (Colombia). ORCID Orjuela-Castro, J.A.: 0000-0001-7857-5064; ORCID Morales-Aguilar, F.S.: 0000-0002-1154-2070; ORCID Mejía-Flórez, L.F.: 0000-0001-6989-6405

2 Autor para correspondencia. jaorjuelac@unal.edu.co
} 
supply chain in Cundinamarca-Bogota. Using a dynamic model system, the current structure was evaluated in comparison with Lean and Agile supply chains. The structures were measured through the performance indicators of quality, responsiveness, logistic operations and costs. This study showed that the Lean and Agile supply chains improved the performance of the current chain in all cases although not for all the involved actors. However, there was a trade-off that indicated a need for a future evaluation of Agile-Lean structures for perishable fruit chains.

Additional key words: fruit supply chain, structure, systems dynamics application, perishability, mango, Cundinamarca-Colombia.

Fecha de recepción: 26-03-2017 Aprobado para publicación: 30-09-2017

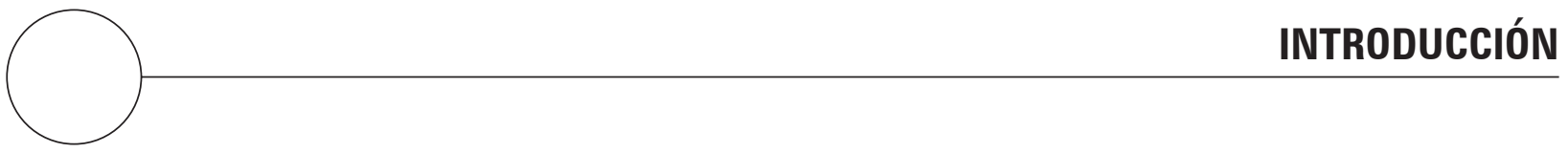

La estructura de la cadena de suministro (CS) define sus procesos y la función que cumple cada eslabón. La estructura es un sistema de componentes que se relacionan entre si y determinan el comportamiento global (Giachetti et al., 2003). Es posible encontrar la influencia entre estrategia y estructura. La importancia de elegir una estrategia para la CS teniendo en cuenta la relación entre el tipo de producto y de cadena, fue planteada por Fisher (1997), quien estableció dos tipos, una con proceso eficiente para productos funcionales y otra con proceso sensible en innovadores; la primera tiene ciclos de vida (CV) largos y demanda estable, la segunda CV cortos y demanda altamente impredecible. Lee (2002) planteó cuatro estructuras para las CS: eficiente, ágil, con capacidad de respuesta y cobertura para el riesgo, mientras Gattorna (2009) señala cuatro tipos: lean, ágil, totalmente flexible y de reposición continua. Los autores Naylor et al. (1999), Agarwal et al. (2006), Naim y Gosling (2011) y Cozzolino et al. (2012) han estudiado la estructura lean y ágil combinadas, con el fin de mejorar el desempeño de la CS, disminuir el efecto látigo y acercarse a la demanda.

La selección adecuada de la estructura de la cadena de suministro (ECS) permite mejorar la calidad, el desempeño logístico, la capacidad de respuesta, la eficiencia y el cumplimiento de la demanda (Aramyan et al., 2006). La ECS de alimentos perecederos (AP) son poco estudiadas y deben contemplar características biofísicas y organolépticas, el tiempo de producción, condiciones de transporte y almacenamiento, su vida útil para mejorar su eficiencia (Lyons y Ma'aram, 2014).
Las cadenas de suministros de alimentos perecederos (CSAP) requieren de medidas de desempeño específicas, una propuesta sobre taxonomía para la evaluación del desempeño logístico en la CSAP se plantea en Orjuela et al. (2006), Ruiz et al. (2015) y Aramyan et al. (2006).

\section{Características de las estructuras}

En la estructura Lean se logra un costo bajo al asegurar que los clientes no tengan exceso de servicio o sobre-atendidos. Los costos reducidos se consiguen haciendo básicos los procesos y eliminar lo que no genere valor (Taylor, 2015).

La estructura ágil presenta una rápida capacidad de respuesta, expedita reconfiguración y niveles de servicio con diferenciación de productos (Mason-Jones y Towill, 1999). Agilidad significa utilizar el conocimiento empresarial y del mercado para aprovechar las oportunidades; gestiona con éxito la demanda cambiante e impredecible en un entorno volátil (Naylor et al., 1999). En este sentido, la logística debe permitir una respuesta rápida, Harrison y Van Hoek (2008) plantean como un objetivo de la logística, asegurar el producto indicado para satisfacer las necesidades exactas del cliente final, entregarlo en el lugar correcto y al momento adecuado. La agilidad requiere de la empresa una manera de actuar, estructuras organizativas, sistemas de información y procesos logísticos (Christopher, 2000).

La CS lean se caracteriza en esencia en la eliminación de residuos, un bajo costo operacional, baja variedad 
y demanda previsible. Los alimentos procesados satisfacen necesidades básicas, son estables, de consumo predecible y largos ciclos de vida, su estabilidad genera competencia lo que deriva en bajos márgenes (Lee, 2002) (Tab. 1).

Por su parte, la CS ágil maneja una rápida reconfiguración y robustez. Empresas con productos perecederos deben manejar ritmos rápidos y regulares en el abastecimiento, Gattona (2009) afirma que la cadena ágil tiene como estrategia combinar la cobertura y capacidad de respuesta (Tab. 1).

\section{Tabla 1. Características de las estructuras de cadena de} suministro (ECS).

\begin{tabular}{|c|c|c|}
\hline Características & Lean & Ágil \\
\hline Tipo de producto & Funcional & Innovador \\
\hline Demanda & Previsible & Volátil \\
\hline $\begin{array}{l}\text { Coordinación de } \\
\text { la CS (compartir } \\
\text { información) }\end{array}$ & $\mathrm{Si}$ & $\mathrm{Si}$ \\
\hline $\begin{array}{l}\text { Eliminación de } \\
\text { residuos }\end{array}$ & Esencial & Deseable \\
\hline $\begin{array}{l}\text { Rápida } \\
\text { reconfiguración }\end{array}$ & Deseable & Esencial \\
\hline Robustez & Indiferente & Esencial \\
\hline Variedad & Bajo & Alto \\
\hline Ciclo de vida & Largo & Corto \\
\hline Costo dominante & Operacional & Comercial \\
\hline Penalización faltantes & $\begin{array}{c}\text { Largo plazo } \\
\text { - contra actual }\end{array}$ & Inmediato-volátil \\
\hline $\begin{array}{l}\text { Mecanismos } \\
\text { pronóstico }\end{array}$ & Algorítmico & Consultivo \\
\hline Estrategia inventario & Mínimo & Significativo \\
\hline $\begin{array}{l}\text { Política de } \\
\text { inventario-producto }\end{array}$ & Terminados & Semielaborados \\
\hline $\begin{array}{l}\text { Selección } \\
\text { proveedores }\end{array}$ & $\begin{array}{c}\text { Costo, confianza } \\
\text { y calidad }\end{array}$ & $\begin{array}{c}\text { Velocidad, flexibilidad } \\
\text { y calidad }\end{array}$ \\
\hline Base proveedores & Pequeño & Grande \\
\hline
\end{tabular}

\section{Medidas de desempeño para las estructuras de las cadenas de suministros}

Teniendo en cuenta las características propias de la cadena de suministro de frutas (CSF) así como de CS lean y ágil, se definió una clasificación de cinco medidas de desempeño: generales, calidad, desempeño logístico, capacidad de respuesta y costos (Tab. 2).

\begin{tabular}{|c|c|c|c|}
\hline \multicolumn{2}{|c|}{ Medidas de Desempeño } & Lean & Ágil \\
\hline \multirow{3}{*}{ Generales } & Satisfacción clientes & medio & muy alto \\
\hline & Tiempo almacenamiento & bajo & bajo \\
\hline & Satisfacción consumidor & medio & muy alto \\
\hline \multirow{5}{*}{ Calidad } & Selección proveedores & alto & alto \\
\hline & Calidad materia prima & alto & alto \\
\hline & Proceso transformación & alto & alto \\
\hline & $\begin{array}{l}\text { Características } \\
\text { biofísicas-organolépticas }\end{array}$ & alto & alto \\
\hline & Perdida inocuidad & bajo & bajo \\
\hline \multirow{3}{*}{$\begin{array}{l}\text { Desempeño } \\
\text { Logístico }\end{array}$} & Nivel inventarios & bajo & alto \\
\hline & Tiempo transporte & medio & bajo \\
\hline & $\begin{array}{l}\text { Tasa pérdidas operaciones } \\
\text { logísticas }\end{array}$ & bajo & alto \\
\hline \multirow{2}{*}{$\begin{array}{l}\text { Capacidad de } \\
\text { Respuesta }\end{array}$} & Tiempo aprovisionamiento & medio & bajo \\
\hline & Cumplimiento entregas & medio & alto \\
\hline \multirow{5}{*}{ Eficiencia } & $\begin{array}{l}\text { Costo } \\
\text { mantenimiento-inventario }\end{array}$ & bajo & alto \\
\hline & Costo transporte & bajo & alto \\
\hline & Costo alistamiento & bajo & alto \\
\hline & Costo adquisición & bajo & alto \\
\hline & $\begin{array}{l}\text { Costo decaimiento } \\
\text { (pérdidas) }\end{array}$ & bajo & alto \\
\hline
\end{tabular}

Fuente: Orjuela et al. (2006); Aramyan et al. (2006); Ruiz et al. (2015); Orjuela-Castro et al. (2016c).

Este trabajo se centra en la CS de mango (CSM), incorpora la pérdida de características físicas y organolépticas, derivados del ciclo de vida y por operaciones logísticas. Algunos autores que han contemplado estas características evalúan la calidad en las CS de alimentos, además del tiempo de conservación del producto (Bourlakis et al., 2012; Orjuela-Castro et al., 2016a). Lean se enfoca en costo, confianza y calidad de los proveedores (Taylor, 2015), mientras la ágil se centra en la velocidad, flexibilidad y calidad (Lehtinen y Torkko, 2005). Adicional a esto, la calidad inicia con el proceso de selección de proveedores, que se basa en la calidad de los suministros y los tiempos de entrega del proveedor.

En las medidas de desempeño logístico, Manzouri et al. (2013) señalan que los niveles de inventario para lean deben ser mínimos, mientras que, en ágil los niveles de inventario son altos lo que permite responder a las variaciones de la demanda (Lehtinen et al., 2005). 
En la estructura ágil es importante la capacidad de respuesta (Christopher y Towill, 2002) con tiempos bajos de aprovisionamiento para cumplir las exigencias de los clientes (Naylor, et al., 1999), a su vez, responder a los cambios en la demanda genera costos más altos. Por su parte, Lean se encarga de alcanzar la eficiencia a través de la reducción los costos (Manzouri et al., 2015).

\section{Generalidades del mango}

Las propiedades organolépticas del mango lo hacen atractivo al consumidor gracias al contenido de azucares, ácidos, aromas y sus pigmentos. La cosecha se realiza cuando se encuentra fisiológicamente maduro, se ha desarrollado y crecido lo suficiente; buscando obtener sus aromas y sabores característicos. Quintero et al. (2013) estudian los cambios a través del tiempo poscosecha de los grados Brix, la textura, el $\mathrm{pH}$ y el porcentaje de acidez. Al ser un fruto climatérico se caracteriza por un rápido aumento en la velocidad de respiración (Lizana y Ochagavía, 1988). El mango presenta cuatro fases climatéricas: una preclimatérica donde se encuentra verde, duro y firme; en la climatérica permanece verde; en la fase pico-climatérica presenta cambio de color y ablandamiento de tejidos y en la fase psclimatérica desarrolla el estado óptimo de madurez (Mahecha et al., 1991).

El objetivo de este artículo es analizar el efecto de la estructura lean y ágil en el desempeño de logística de la CSAP aplicando dinámica de sistemas al caso de estudio de la CS de mango en Cundinamarca-Bogotá.

\section{MATERIALES Y MÉTODOS}

Como método se utilizó la simulación continua, bajo el paradigma de dinámica de sistemas, Esto permite establecer el comportamiento de la CSM, el cual fue diseñado en iThink, v. 9.0 (Isee Systems, Lebanon, $\mathrm{NH}$ ). Los datos de entrada se obtuvieron del análisis de la oferta (DANE y Agronet, Colombia), la demanda obtenida de la encuesta ENSIN (2005 y 2010), el comportamiento logístico a partir de la aplicación de encuestas en 2015 a 20 tenderos, 15 en comerciantes de plazas de mercado, siete mayoristas de Corabastos, ocho transportadores y 20 en agricultores. Con el instrumento se determinó la cultura comercial y el manejo logístico del mango por parte de los actores de la CSM, para ver algunos de los resultados consultar Orjuela et al. (2015b). Para establecer las pérdidas por ciclo de vida del mango, nos apoyamos en el comportamiento poscosecha.

\section{Modelo en dinámica de sistemas}

En el modelo de dinámica de sistemas, se representan las variables de nivel (acumuladores) y de flujo (tasas), los flujos crecen o disminuyen los niveles a través del tiempo. Existen varios tipos de flujos, para las CS (Forrester, 1961), en CS alimentarias son importantes los de material (frutas frescas, procesadas y las pérdidas) y de información (pedidos y costos). Se estableció la dinámica del sistema mediante ciclos de retroalimentación en forma de diagramas causales, los cuales pueden ser de refuerzo (positivos) o balanceadores (negativos) (Sterman, 2000). La incidencia de la estructura en el desempeño de logístico de la CSM, permitió plantear la siguiente hipótesis: "Una correcta estructura lean o ágil en la CSM mejora su desempeño en calidad, capacidad de respuesta, desempeño logístico y eficiencia, para los diferentes actores y para toda la cadena". Se presentan cuatro diagramas causales, en concordancia con las medidas de desempeño descritas (Tab. 2).

En el causal de calidad, se presentan tres ciclos balanceadores (Fig. 1), ya que son ciclos de equilibrio o balanceo con efecto negativo entre variables (B), en cuanto a los aspectos asociados a la influencia de la fruta en la pérdida de inocuidad.

En el causal de capacidad de respuesta (Fig. 2), la naturaleza del ciclo 5 depende de la estructura de la

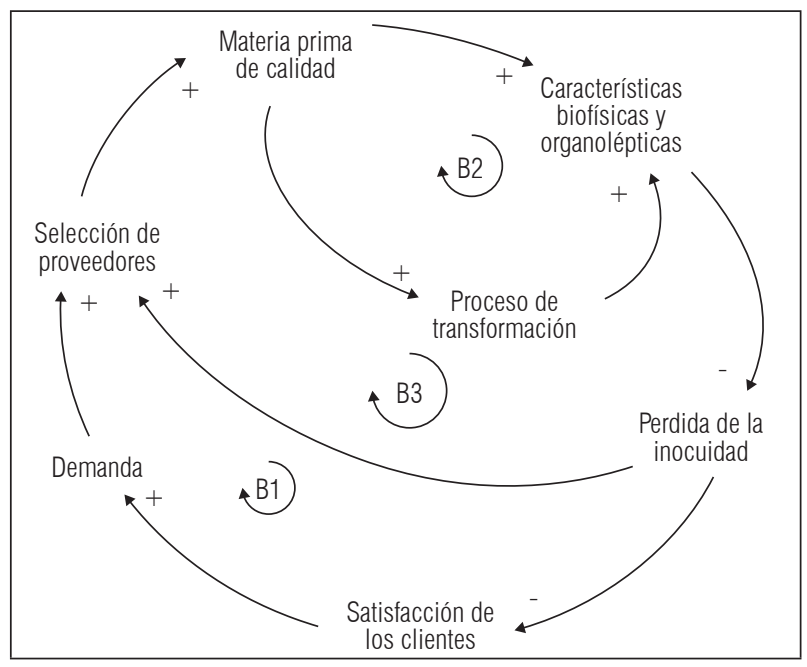

Figura 1. Causal de calidad propuesto para la cadena de suministro de mango en Cundinamarca, Colombia. 


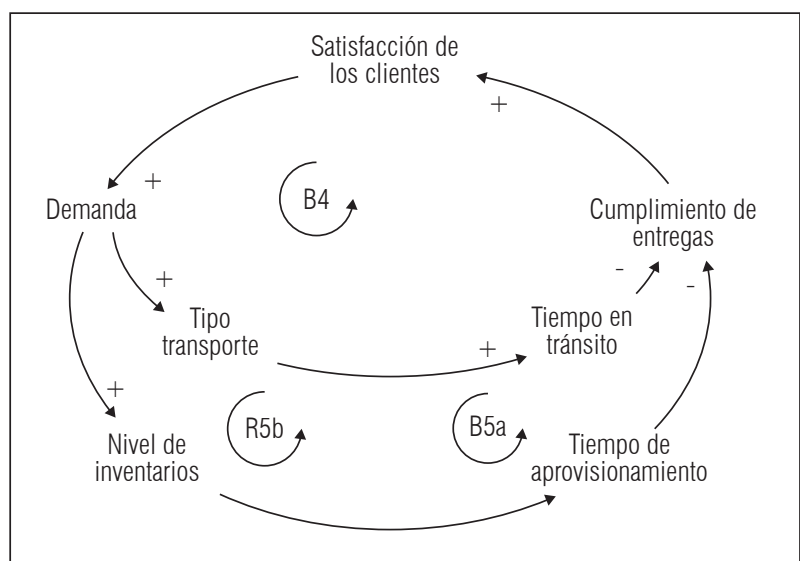

Figura 2. Causal para capacidad de respuesta de la cadena de suministro del mango en Cundinamarca, Colombia.

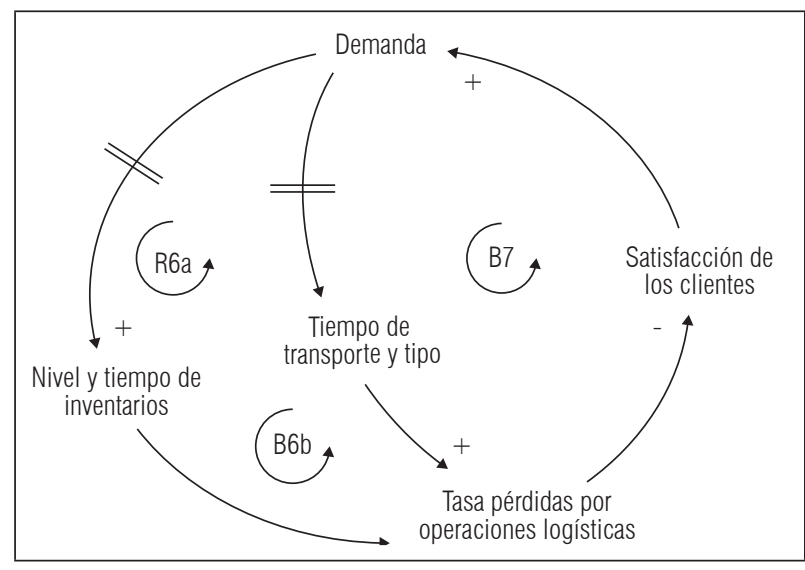

Figura 3. Causal desempeño logístico de la cadena de suministro del mango en Cundinamarca, Colombia. cadena, para la estructura Agíl es un ciclo reforzador refuerzo con efecto positivo entre variables (R5b), esto es causado por el manejo del inventario, ya que a mayor nivel disminuye el tiempo de aprovisionamiento, mejorando el cumplimiento de entregas; mientras que para la estructura Lean es balanceador (B5a), pues el bajo inventario incide negativamente en la entrega. Por su parte, el ciclo B4 es negativo dado que a mayor tiempo de transito menor cumplimiento en entregas.

El causal para el desempeño logístico (Fig. 3) presenta el ciclo 6 que para estructura lean es balanceador (B6b), ya que el arco - nivel de inventarios - tasa de pérdidas es negativo, mientras en la ágil el arco es positivo, reforzador (R6a), así mismo tiene un ciclo balanceador B4, dado que al crecer el tiempo de transito incide negativamente en el cumplimiento de entregas.

El causal de costos, presenta cuatro ciclos balanceadores $\mathrm{B} 7, \mathrm{~B} 8, \mathrm{~B} 9$ y B10 dado que a mayor costo menos eficiencia, no obstante presenta un ciclo reforzador, el derivado del proceso de transformación (Fig. 4). Se resalta como este ciclo incluye todas las pérdidas y su incidencia en costos.

A partir de los diagramas causales y de la información obtenida de encuestas e información secundaria se realizó el modelo en Forrester (Fig. 5), desarrollado como una evolución de los modelos creados por Orjuela y Herrera (2014), Orjuela et al. (2015a), Orjuela-Castro et al. (2016a) y Orjuela-Castro et al. (2016b). Si bien la estructura de Forrester fue la misma utilizada en los trabajos mencionados, en este se modificó el modelo, al obtener una estructura que permite la medición de la calidad, el desempeño logístico, la

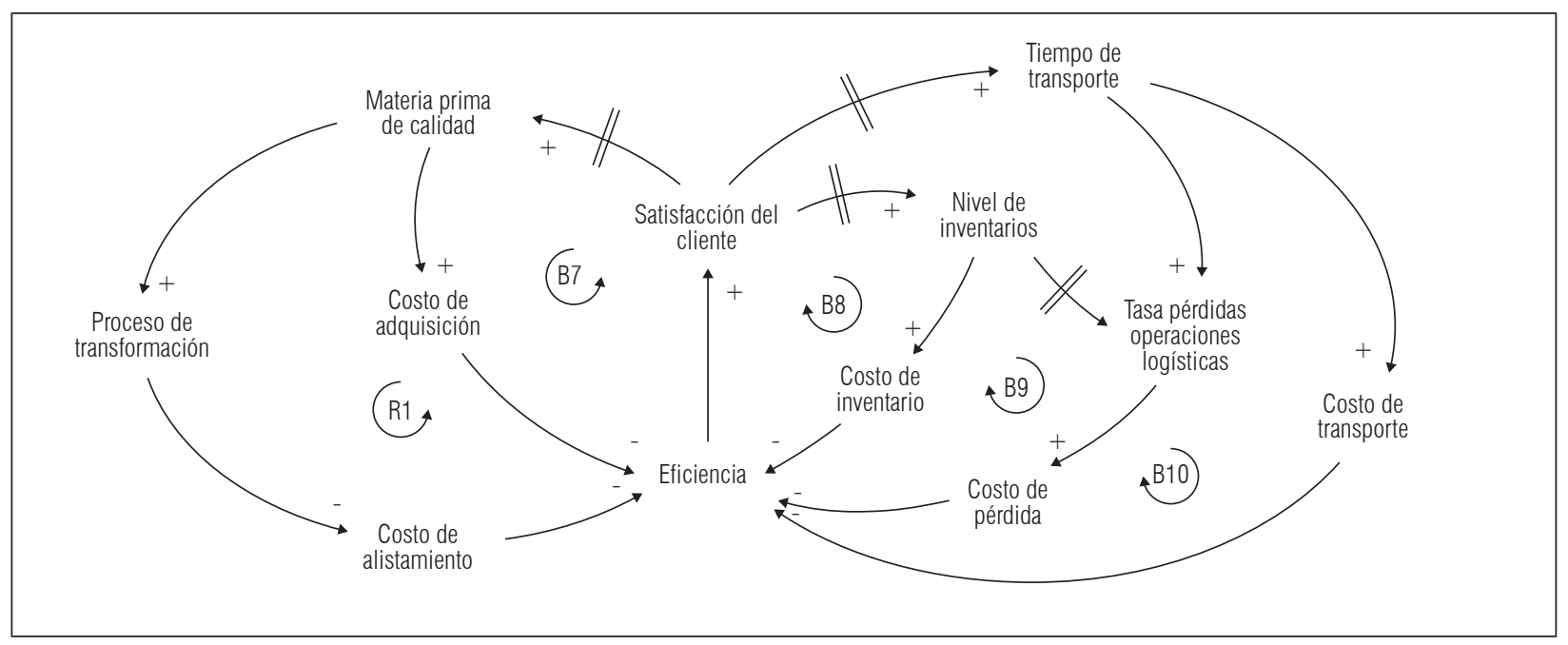

Figura 4. Ciclo de realimentación de costos de la cadena de suministro del mango en Cundinamarca, Colombia. 
capacidad de respuesta y los costos, teniendo como variable el ajuste de producción, el tipo de vehículo, utilización de las máquinas (en agroindustria) y el ajuste del tiempo de procesamiento de orden.

En la figura 5 se presentan cada uno de los actores incluidos en el modelo, los agricultores, el distribuidor (intermediario) de fruta fresca, la agroindustria, el distribuidor (intermediario) de producto procesado y los puntos de venta de fruta fresca o procesada. Las líneas azules representan el flujo de material entre los actores y las rojas el flujo de información. El productor le puede vender al intermediario (distribuidor de fruta fresca) o a la industria directamente, la agroindustria le vende la fruta procesada al intermediario (distribuidor de fruta procesada) o directamente a los puntos de venta, así mismo el distribuidor de fruta fresca le puede vender directamente a los puntos de venta. LA información entre ellos se refiere a los pedidos colocados por los actores agua debajo de la cadena.

La estructura del modelo varía con base en las siguientes variables:

- ajuste de rendimiento de producción de fruta en el agro,

- tipo de vehículo para el agricultor y el distribuidor,

- ajuste del flujo de producción y

- del procesamiento de orden.

Estas variaciones no se ven reflejadas en la estructura del modelo de Forrester (Fig. 5).

Producción: se modificó el rendimiento $\left(\mathrm{t} \mathrm{h} \mathrm{a}^{-1}\right)$ con base en estadísticas de 2007-2015, se asumió normalidad. Se incrementa en un 16\% la producción en la cadena ágil y se disminuye en un 16\% para la cadena lean, estos porcentajes fueron hallados con base en los límites inferior y superior de la distribución normal con un porcentaje de confiabilidad del $95 \%$.

Tipos de vehículos: se contemplaron tres tipos de vehículos para el transporte departamental y urbano, con diferencias en velocidad, tiempo y costos por trayecto. Los tiempos de tránsito, carga y descarga se ingresaron como demora en la tasa de llegada. La capacidad de vehículos de tipo 1, 2 y 3 fueron de 1,5 y $10 \mathrm{t}$, respectivamente.

Flujo de producción: fue ajustado mediante la utilización de las máquinas en agroindustria, se cambian tiempos de alistamiento en el proceso de transformación, lo cual influye en tiempos de aprovisionamiento y niveles de inventario para fruta fresca y procesada. Las máquinas trabajan 2 turnos para la cadena actual, un turno en lean y tres en ágil. Con base en esto, se toma los 2 turnos como el 100\%, 1 turno como el $50 \%$ y 3 turnos como $150 \%$ de la utilización de las máquinas.

Procesamiento de orden: la variable permite el ajuste del tiempo de pedidos, a la cadena actual se asignó 1 (100\%), para lean 1,5 y ágil 0,5. Se incluyó una demora en la tasa de entrega.

\section{Escenarios para las estructuras de la cadena de suministro}

Con el propósito de establecer la estructura en la CSM en Cundinamarca-Bogotá, se evaluaron 3 escenarios la cadena actual, ágil y lean (Tab. 3).

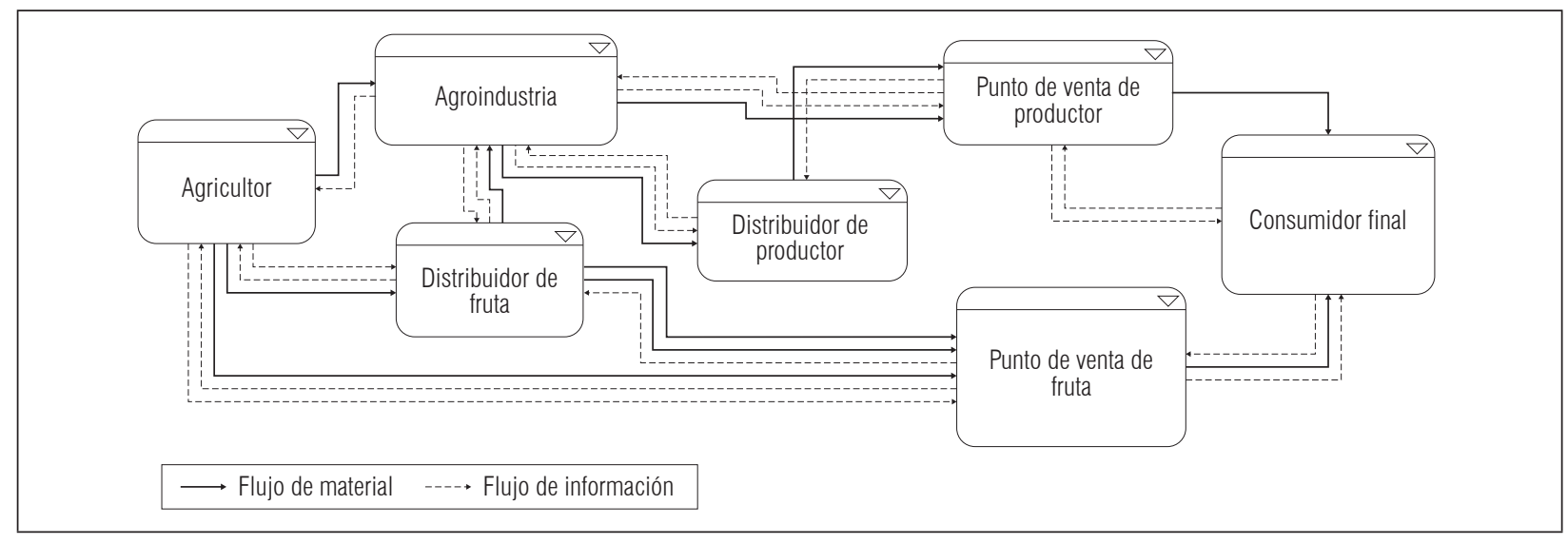

Figura 5. Modelo Forrester de la cadena de suministro del mango en Cundinamarca, Colombia. 
Tabla 3. Variables que modifican los escenarios de la cadena de suministro de mango en Cundinamarca, Colombia.

\begin{tabular}{|l|c|c|c|}
\hline \multirow{2}{*}{\multicolumn{2}{|c|}{ Variables }} & \multicolumn{3}{|c|}{ Escenarios: estructuras } \\
\cline { 2 - 4 } & Actual & Ágil & Lean \\
\hline Ajuste de producción & 1 & 1,16 & 0,84 \\
\hline Tipos vehículo & 2 & 1,00 & 3,00 \\
\hline Utilización máquina & 1 & 1,50 & 0,50 \\
\hline Procesamiento orden & 1 & 0,50 & 2,00 \\
\hline
\end{tabular}

\section{Escenario 1}

CS actual. El agricultor envía toda la cosecha disponible a los eslabones mayorista, agroindustria o punto de venta, las ordenes aguas abajo son determinadas por el nivel de inventario disponible, la producción actual es de 10,07 ( $\left.\mathrm{t} \mathrm{ha} \mathrm{a}^{-1}\right)$. El mango es transportado en camiones de estacas de dos ejes, desde el agricultor a los demás eslabones, teniendo en cuenta la temperatura en la cual debe ser transportado. Estos vehículos cuentan con capacidad de $5 \mathrm{t}$ y una velocidad departamental de $37 \mathrm{~km} \mathrm{~h}^{-1}$ promedio y urbana de 14 $\mathrm{km} \mathrm{h}^{-1}$. El porcentaje de utilización de las máquinas y el ajuste de procesamiento de orden es del 100\%. Las pérdidas en la CSM son del 4,57\%.

\section{Escenario 2}

CS ágil. Se incrementó un 16\% sobre la producción actual, se mejoró la capacidad de respuesta con inventarios altos y se modificó el vehículo a uno de $1 \mathrm{t}$ con mayor velocidad. La capacidad de producción en agroindustria aumentó un 50\%, para responder a la demanda inesperada, el incremento se hizo en turnos laborales u horas extra. El tiempo de procesamiento de orden se redujo en un 50\% respecto a la CS actual, para una respuesta rápida, el tiempo se disminuyó manejando líneas de preparación de pedidos en paralelo.

\section{Escenario 3}

CS lean. La producción se disminuyó $16 \%$ con respecto a la CS actual, con el propósito de disminuir pérdidas mediante inventarios bajos, bajar los costos y aumentar la eficiencia. Por ello, se seleccionó un vehículo con mayor capacidad del escenario 1, permitiendo disminuir la cantidad de viajes y los costos de transporte. La capacidad de producción en agroindustria se disminuyó un $50 \%$, el nivel y costos del inventario. El tiempo de procesamiento de orden aumentó en $100 \%$, un proceso de preparación de pedidos secuencial, permitió acumular pedidos y disminuir costos de entrega.

Con base en la valoración cualitativa (Tab. 2), se definen las medidas y unidades de desempeño (Tab. 4) que corresponden a los cuatro ciclos denotas a lo largo del artículo (calidad, desempeño logístico, capacidad de respuesta y costos).

Se realizaron cinco simulaciones por cada escenario (Yasarcan, 2011), en cada simulación se usó un valor semilla diferente (Hudnurkar y Rathod, 2012). El tiempo de simulación fue 3,650 d, con un DT $=0,25$ (intervalo de tiempo en simulación). Por cada medida de desempeño se comparan la CS actual, lean y ágil, para los eslabones, toda la CSM y los canales de distribución propuestos, los cuales son: (Canal C1) agricultor - distribuidor de fruta - punto de venta de fruta; (Canal C2) agricultor - agroindustria - punto de venta; (Canal C3) agricultor - distribuidor de fruta - agroindustria.

Tabla 4. Medidas de desempeño para la cadena de suministro del mango en Cundinamarca, Colombia; modelo.

\begin{tabular}{|c|c|c|}
\hline \multicolumn{2}{|r|}{ Medida } & Unidad \\
\hline \multirow[t]{2}{*}{ Calidad } & $\begin{array}{l}\text { Características biofísicas, } \\
\text { organolépticas }\end{array}$ & $\begin{array}{l}\text { Color, }{ }^{\circ} \text { Brix, pH, } \\
\% \text { acidez, textura } \\
\text { de la cáscara }\end{array}$ \\
\hline & Pérdidas promedio & \\
\hline \multirow{2}{*}{$\begin{array}{l}\text { Desempeño } \\
\text { logístico }\end{array}$} & Inventario promedio & $t d^{-1}$ \\
\hline & Transporte promedio & $t d^{-1}$ \\
\hline \multirow{2}{*}{$\begin{array}{l}\text { Capacidad de } \\
\text { respuesta }\end{array}$} & Tiempo aprovisionamiento & Días (d) \\
\hline & Cumplimiento entregas & \\
\hline Costos & $\begin{array}{l}\text { Costos logísticos, } \\
\text { adquisición y producción }\end{array}$ & Pesos colombianos \\
\hline
\end{tabular}

\section{RESULTADOS Y DISCUSIÓN}

A continuación se presentan los resultados obtenidos del modelo de dinámica de sistemas, teniendo en cuenta los ciclos de análisis y las medidas de desempeño.

\section{Calidad}

Las características biofísicas y organolépticas: ${ }^{\circ}$ Brix, $\mathrm{pH}, \%$ acidez y textura, se determinaron a partir del 
estudio de Quintero et al. (2013) y teniendo en cuenta el tiempo total del mango en los canales de distribución obtenidos de la simulación (Fig. 6).

Los C1 y C2 llegan en el periodo climatérico en los tres escenarios (Fig. 6), Quintero et al. (2013) encontraron que el mango llega en la fase climatérica en 6 d. En el C1, para lean, la fruta tarda 5,85 d del agricultor al punto de venta; mientras en el C3, en lean $y$ actual, el mango llega a la agroindustria en el pico climatérico. Los valores calculados para ${ }^{\circ}$ Brix, textura de la cascara, $\mathrm{pH}$ y porcentaje de acidez (Tab. 5) fueron basados en Quintero et al. (2013).
En la estructura lean el C1 presenta mayor concentración de sólidos solubles con $17,372{ }^{\circ}$ Brix, en la ágil, el tiempo de duración en el C1 de 5,39 d y permite que el mango llegue al punto de venta con menor maduración, el canal 2 presenta en promedio 3,61 d y 5,82 d para el C3, esto se encuentra relacionado con la capacidad de respuesta, disminución del tiempo en ajuste de procesamiento de orden y la selección de vehículos más veloces. En el $\mathrm{C} 1$ para lean el pH es mayor respecto a ágil, con un $\mathrm{pH}$ de 4,536. Contrario a esto, el porcentaje de acidez en lean es menor que en ágil, relación inversa entre $\mathrm{pH}$ y porcentaje de acidez, evidencia una disminución de ácidos orgánicos

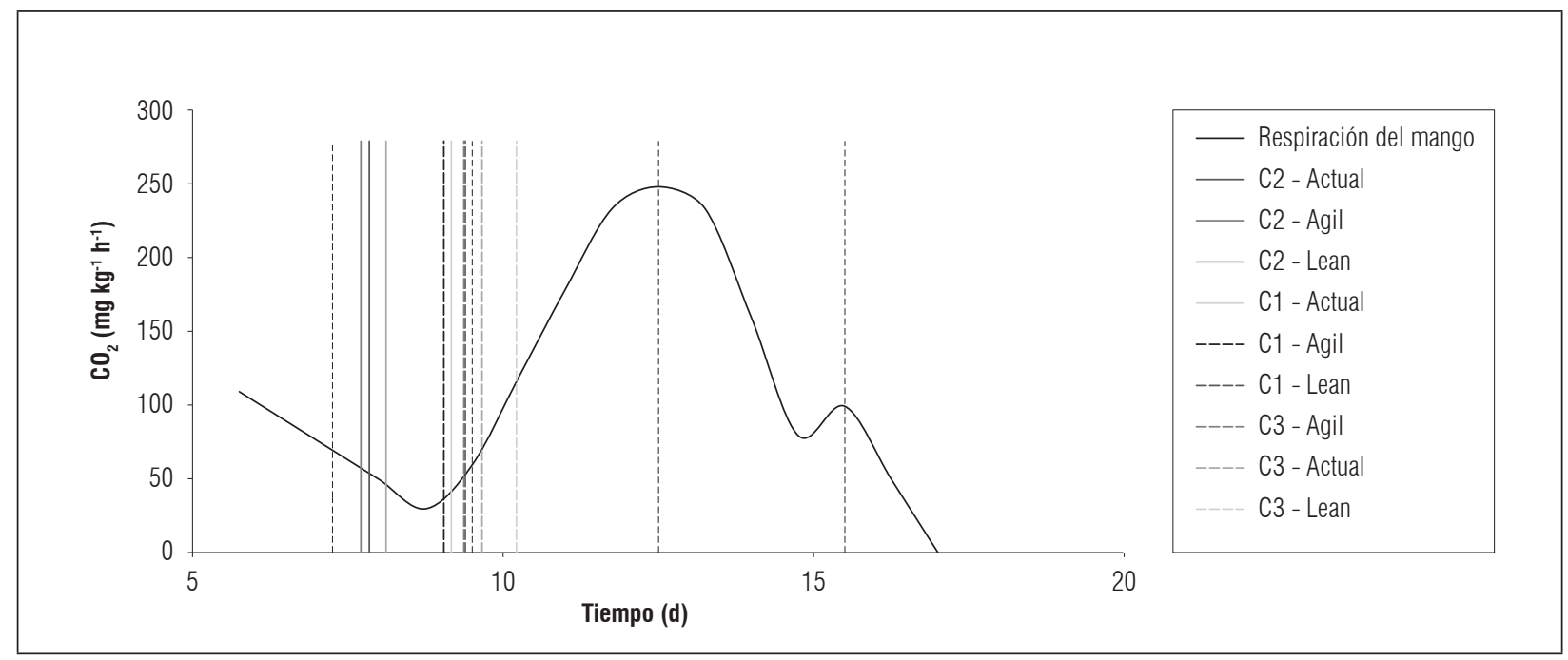

Figura 6. Resultados de la maduración del mango (respiración del fruto) según canal de distribución en la cadena de suministro de Cundinamarca, Colombia.

Tabla 5. Resultados características biofísicas.

\begin{tabular}{|c|c|c|c|c|c|}
\hline \multirow[b]{2}{*}{ Escenario } & \multicolumn{5}{|c|}{ Características del mango } \\
\hline & Días & ${ }^{\circ}$ Brix & $\mathrm{pH}$ & Acidez (\%) & $\begin{array}{l}\text { Textura cascara } \\
\text { (kg fuerza) }\end{array}$ \\
\hline C1 - Actual & 5,55 & 17,145 & 4,467 & 0,683 & 3,912 \\
\hline C1 - Ágil & 5,39 & 17,020 & 4,430 & 0,704 & 3,955 \\
\hline C1 - Lean & 5,85 & 17,372 & 4,536 & 0,645 & 3,831 \\
\hline C2 - Actual & 3,79 & 15,585 & 4,062 & 0,932 & 4,387 \\
\hline C2 - Ágil & 3,61 & 15,397 & 4,020 & 0,961 & 4,435 \\
\hline C2 - Lean & 4,15 & 15,943 & 4,145 & 0,877 & 4,290 \\
\hline C3 - Actual & 6,21 & 17,633 & 4,618 & 0,600 & 3,733 \\
\hline C3 - Ágil & 5,82 & 17,349 & 4,529 & 0,648 & 3,839 \\
\hline C3 - Lean & 6,95 & 18,136 & 4,789 & 0,512 & 3,534 \\
\hline
\end{tabular}


al aumentar el tiempo de entrega al punto de venta. Según los datos de textura de la cascara, cuanto más largo sea el tiempo de entrega, menor es la fuerza necesaria para la ruptura de la misma, epidermis más blanda. El comportamiento de las características fisicoquímicas en los C2 y C3 es similar al descrito para C1 (Tab. 5). Este comportamiento está en concordancia con Quintero et al. (2013).

\section{Pérdidas por operaciones logísticas}

La CSM lean presentó menos pérdidas (Tab. 6), al disminuir el 39\% en inventario y $38 \%$ en transporte. Los eslabones más favorecidos son el agricultor y el distribuidor, con 29 y $41 \%$, respectivamente, mientras la agroindustria y punto de venta presentaron un aumento del 2\%. La CSM ágil mostró un aumento en pérdidas de $21 \%$ en almacenamiento y $20 \%$ en transporte, aunque el eslabón agroindustria se favorece al disminuir en $1 \%$ en almacenamiento y el distribuidor $0,8 \%$ en transporte. Todos estos porcentajes se dan respecto a la cadena actual. De manera agregada, el porcentaje de pérdida de fruta para el escenario actual es el 7,06\% del total de mango, en ágil el 7,11\% y en lean el 6,98\% del mango total en inventario y transporte. En este último, se podría evaluar el modo de transporte y su incidencia en las pérdidas de la CSM, estudiado por Orjuela-Castro et al. (2016b) en otras frutas, también el efecto de la infraestructura de la ciudad, planteado por Orjuela et al. (2015a).

\section{Desempeño logístico}

Para visualizar las medidas de desempeño logístico se calculó el inventario y transporte de mango fresco y pulpa a lo largo de la cadena y en cada eslabón.

\section{Inventario}

La CSM actual maneja en promedio 207,7 t en inventario de fruta fresca, aumenta hasta en un $123,9 \%$ en promedio para ágil y disminuye en un 35,5\% para lean. El inventario de pulpa de mango aumenta en un $15,5 \%$ en promedio en ágil, mientras que en lean disminuye en un $2,4 \%$. Los bajos inventarios en alimentos y altos en ágil son planteados por Giachetti et al. (2003), modelamos los mismos efectos de este estudio.

Para todos los eslabones el menor inventario se presenta en la CSM lean, exceptuando el distribuidor de pulpa de mango, en cuyo caso el menor inventario es el de la CSM actual.

\section{Transporte}

La estructura ágil presenta la mayor cantidad promedio de mango fresco y procesado transportado, con 86,09 y 37,8 t, respectivamente, estos resultados corresponden con los encontrados por Giachetti et al. (2003) en otras cadenas ágiles. El escenario ágil transporta un 3,93 y $5,12 \%$ de mango y pulpa de mango, respectivamente, por encima del escenario actual.

\section{Capacidad de respuesta}

Para medir el tiempo de aprovisionamiento se tomaron los tres canales propuestos, lean lo incrementa en todos los canales de distribución, mientras que ágil lo disminuye hasta en un $44 \%$ en el canal 2 respecto a la CSM actual, en la CSM completa ágil tuvo el menor tiempo de respuesta, mientras que el escenario lean obtuvo el mayor tiempo de aprovisionamiento y procesamiento de orden, resultados similares a los encontrados por Lyons y Ma'aram (2014). En cuanto al cumplimiento de la demanda, se midió para mango y pulpa de mango; ágil tiene el mayor cumplimiento sobre la demanda de fruta fresca, cercano al 100\%, superando en $1,04 \%$ la cadena actual y 3,42\% sobre lean (línea gris en Fig. 7). En la pulpa de mango no se ven variaciones significativas, con un cumplimiento que tiende al 60\% (línea negra en Fig. 7). Este fenómeno había sido estudiado por Orjuela-Castro et al. (2016a), evaluando los efectos de integración externa

Tabla 6. Pérdidas por operación logística (toneladas) para dos cadenas de suministro de mango en Cundinamarca, Colombia.

\begin{tabular}{|c|c|c|c|c|c|c|c|c|c|}
\hline \multirow{2}{*}{ Escenarios } & \multicolumn{5}{|c|}{ Almacén } & \multicolumn{3}{|c|}{ Transporte } & \multirow{2}{*}{$\begin{array}{l}\text { Pérdidas } \\
\text { cadena }\end{array}$} \\
\hline & Agricultor & Distribuidor & Agroindustria & Punto venta & Cadena & Agricultor & Distribuidor & Cadena & \\
\hline Actual & 61.919 & 608.841 & 5.622 & 15.251 & 691.636 & 18.748 & 2.034 & 20.782 & 712.419 \\
\hline Ágil & 73.806 & 73.9650 & 5.551 & 15.210 & 834.220 & 21.763 & 2.019 & 23.781 & 858.001 \\
\hline Lean & 43.822 & 36.0494 & 5.724 & 15.502 & 425.546 & 15.877 & 2.053 & 17.930 & 443.476 \\
\hline
\end{tabular}




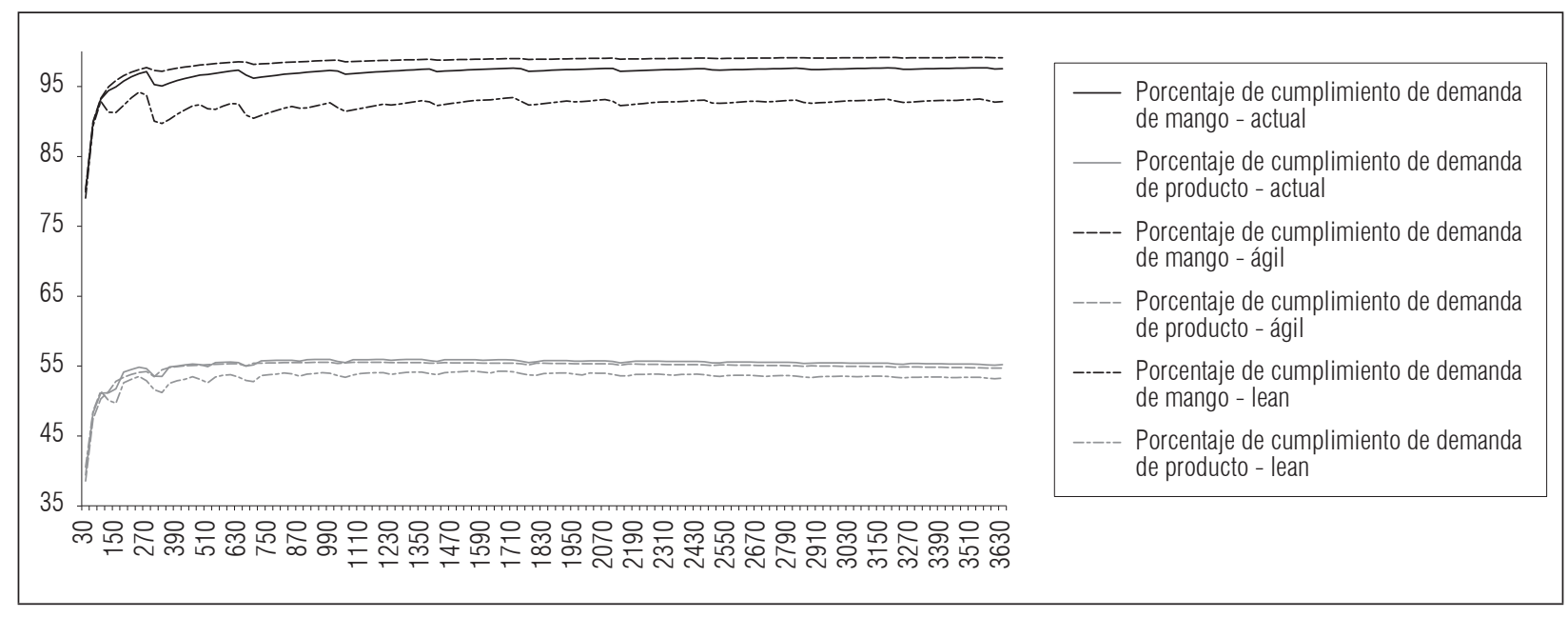

Figura 7. Cumplimiento de la demanda de fruta y pulpa de mango en tres estructuras de la cadena suministro en Cundinamarca, Colombia.

en la CSM. Las diferencias de los tiempos de respuesta entre los escenarios no superan el día, ya que los trayectos en el departamento de Cundinamarca no son extensos.

La gráfica de la figura 7 ha sido sometida a cambios extremos en los parámetros, con el fin de obtener un mejor análisis de sensibilidad.

\section{Costos}

El costo de transporte genera mayor impacto sobre el cambio por escenario. Éste varía dependiendo de la cantidad de vehículos que serán usados por día. Lean disminuye los costos hasta en un 32,39\% con respecto a la cadena actual. Los niveles de inventario y el transporte de carga en la cadena afectaron significativamente sus costos. Dado que el nivel alto de inventario incrementan sus costos y las pérdidas por decaimiento, el escenario ágil obtuvo un aumento significativo de sus costos por encima de los otros escenarios. Por otro lado, el escenario lean apostó por niveles bajos de inventario $y$, teniendo en cuenta que se busca reducir costos, seleccionó vehículos con mayor capacidad de carga y usó estrategias de acumulación de pedido que permitieron disminuir la inversión en estas actividades, aunque se incrementó el tiempo (Tab. 7).

\section{Resultados globales}

Los valores totales al finalizar la experimentación de 10 años evidencia que no existe una estructura que mejore todas las medidas de desempeño estudiadas (Tab. 8), se han resaltado las de mejor desempeño, aparece un problema que muestra enfrentamiento entre diferentes medidas de desempeño u objetivos, comúnmente llamado trade-off.

Lo anterior nos plantea la necesidad de evaluar estructuras mixtas, posiblemente por actores. Este enfoque se ha planteado por Naim y Gosling (2011) y Cozzolino et al. (2012), no obstante, ellos no lo han aplicado a cadenas de alimentos perecederos, ni con las medidas de desempeño estudiadas en esta investigación.

\section{CONCLUSIONES}

El modelo planteado en dinámica de sistemas permitió modelar las estructuras lean, ágil y comparar las medidas de desempeño propuestas vs. la cadena de suministro del mango actual. Fue evaluado el comportamiento de los agentes (eslabones) y de toda la cadena, en cuatro medidas de desempeño, eficiencia, capacidad de respuesta, logística y calidad.

Si bien los resultados encontrados no permiten definir una estructura para la CSM, si muestran que la adopción de cualquiera, lean o ágil, mejoran el desempeño de la cadena actual. No obstante depende de la estrategia definida por los agentes de la cadena, es el caso de los que quiere mayor eficiencia deberán emplear lean, mientras los interesados en una mayor capacidad de respuesta la adecuada es la ágil. En lo que respecta a las pérdidas, la CS ágil las disminuye frente 
Tabla 7. Costos en las diferentes estructuras de la cadena de suministro de mango en Cundinamarca, Colombia.

\begin{tabular}{|l|c|c|c|c|c|c|}
\hline \multicolumn{1}{|c|}{ Escenario } & Adquisición & $\begin{array}{c}\text { Mantenimiento } \\
\text { de inventario }\end{array}$ & Pérdidas & Transporte & Alistamiento & Total \\
\hline Actual & $0 \%$ & $0 \%$ & $0 \%$ & $0 \%$ & $0 \%$ & $0 \%$ \\
\hline Ágil & $12,33 \%$ & $19,90 \%$ & $20,25 \%$ & $400,00 \%$ & $-58,12 \%$ & $20,24 \%$ \\
\hline Lean & $-7,29 \%$ & $-35,47 \%$ & $-32,42 \%$ & $-50,00 \%$ & $176,31 \%$ & $-32,39 \%$ \\
\hline
\end{tabular}

Porcentajes comparados con la CSM actual; (-) significa disminución.

Tabla 8. Resultados finales.

\begin{tabular}{|l|c|c|c|c|}
\hline \multicolumn{1}{|c|}{ Escenario } & $\begin{array}{c}\text { Características biofísicas y } \\
\text { organolépticas (d) }\end{array}$ & $\begin{array}{c}\text { Pérdidas promedio } \\
\text { de mango }(\mathrm{t})\end{array}$ & $\begin{array}{c}\text { Inventario promedio } \\
\text { de mango (t) }\end{array}$ & $\begin{array}{c}\text { Transporte promedio } \\
\text { de mango (t) }\end{array}$ \\
\hline Actual & 15,55 & $712.418,53$ & 266,92 & 118,86 \\
\hline Ágil & 14,82 & $858.001,04$ & 362,6 & 123,89 \\
\hline Lean & 16,95 & $443.475,54$ & 189,88 & 95,08 \\
\hline \multicolumn{1}{|c|}{ Escenario } & Costos (\%) & Tiempo de aprovisionamiento (d) & Cumplimiento de entregas (\%) \\
\hline Actual & $0 \%$ & 2,45 & $76,42 \%$ \\
\hline Ágil & $20,24 \%$ & 1,54 & $76,69 \%$ \\
\hline Lean & $-32,39 \%$ & 4,26 & $74,74 \%$ \\
\hline
\end{tabular}

a las derivadas de la perecibilidad, mientras la CS lean respecto a las generadas por la operación logística.

Los trade-off encontrados en este estudio, plantean la necesidad de evaluar cadenas combinadas ágil-lean.

Conflicto de intereses: el manuscrito fue preparado y revisado con la participación de los autores, quienes declaran no tener algún conflicto de interés que coloquen en riesgo la validez de los resultados aquí presentados.

\section{REFERENCIAS BIBLIOGRÁFICAS}

Agarwal, A., R. Shankar y M.K. Tiwari. 2006. Modeling the metrics of lean, agile and leagile supply chain: An ANP-based approach. Eur. J. Oper. Res. 173(1), 211225. Doi: 10.1016/j.ejor.2004.12.005

Aramyan, L., C. Ondersteijn, O. Van Kooten y A. Lansink. 2006. Performance indicators in agri-food production chains. pp. 47-64. En: Ondersteijn, C.J.M., J.H.M. Wijnands, R.B.M. Huirne y O. van-Kooten (eds.). Quantifying the agri-food supply chain. Springer, Wageningen, The Netherlands. Doi: 10.1007/1-4020-4693-6_5

Bourlakis, M., G. Maglaras y C. Fotopou. 2012. Creating a "best value supply chain"? Empirical evidence from the Greek food chain. Int. J. Logist. Manag. 23(3), 360382. Doi: 10.1108/09574091211289228
Christopher, M. 2000. The agile supply chain competing in volatile markets. Ind. Market. Manag. 29, 37-44. Doi: 10.1016/S0019-8501(99)00110-8

Christopher, M. y D.R. Towill. 2002. Developing market specific supply chain strategies. Int. J. Logist. Manag. 13(1), 1-14. Doi: 10.1108/09574090210806324

Cozzolino, A., S. Rossi y A. Conforti. 2012. Agile and lean principles in the humanitarian supply chain: The case of the United Nations World Food Programme. J. Humanit. Logist. Supply Chain Manag. 1(2), 16-33. Doi: 10.1108/20426741211225984

Fisher, M. 1997. What is the right supply chain for your product? Harvard Bus. Rev. 75, 105-116.

Forrester, J. 1961. Industrial dynamics. Productivity Press, Portland, OR, USA.

Gattorna, J. 2009. Cadenas de abastecimiento dinámicas. ECOE Ediciones, Bogotá.

Giachetti, R., L. Martinez, O. Sáenz y C. Chin-Seng, 2003. Analysis of the structural measures of flexibility and agility using a measurement theoretical framework. Int. J. Prod. Econ. 47-62. Doi: 10.1016/ S0925-5273(03)00004-5

Harrison, A. y R. Van Hoek. 2008. Logistics management and strategy. $3^{\text {rd }}$ ed. Pearson. Harlow, UK.

Hudnurkar, M. y U. Rathod. 2012. Collaborative supply chain: insights from simulation. Int. J. Syst. Assur. Eng. Manag. 3(2), 122-144. Doi: 10.1007/ s13198-012-0114-9 
Lee, H.L. 2002. Aligning supply chain strategies with product uncertainties. Calif. Manag. Rev. 44(3), 105-119. Doi: $10.2307 / 41166135$

Lehtinen, U. y M. Torkko. 2005. The lean concept in the food inductry: A case of contract a manufacturer. J. Food Distribution Res. 36, 57-67.

Lizana, A. y A. Ochagavía. 1988. Maduración, temperaturas de almacenaje y atmósfera controlada en mangos. Idesia 15, 77-83.

Lyons, A.C. y A. Ma'aram. 2014. An examination of multi-tier supply chain strategy alignment in the food industry. Int. J. Prod. Res. 527, 911-1925. Doi: 10.1080/00207543.2013.787172

Mahecha, G., L.A. de-Civetta y C. Rodríguez. 1988. Normas de calidad para las variedades de mango 'Tommy Atkins' y 'Común' (Hilacha). Rev. Colomb. Quim. 20(2), 10-17.

Manzouri, M., M.N.A. Rahman, N. Saibani, C.R.M. Zain. 2013. Lean supply chain practices in the Halal food. Int. J. Lean Six Sigma 4(4), 389-408. Doi: 10.1108/ IJLSS-10-2012-0011

Mason-Jones, R. y D.R. Towill. 1999. Total cycle time compression and the agile supply chain. Int. J. Prod. Econ. 62(1), 61-73. Doi: 10.1016/S0925-5273(98)00221-7

Naim, M.M. y J. Gosling. 2011. On leanness, agility and leagile supple chains. Int. J. Prod. Econ. 131(1), 342-354. Doi: 10.1016/j.ijpe.2010.04.045

Naylor, J.B., M.M. Naim y D. Berry. 1999. Leagility: Integrating the lean and agile manufacturing paradigms in the total supply chain. Int. J. Prod. Econ. 62(1-2), 107-118. Doi: 10.1016/S0925-5273(98)00223-0

Orjuela, J.A., M.E. Calderón y S.P. Buitrago. 2006. La cadena agroindustrial de frutas, uchuva y tomate de árbol. Universidad Distrital Francisco José de Caldas, Bogotá, Colombia.

Orjuela, J.A., W. Casilimas y M.M. Herrera. 2015a. Impact analysis of transport capacity and food safety in Bogota. pp. 1-7. En: Engineering Applications - International Congress on Engineering, Bogota, Colombia. Doi: 10.1109/WEA.2015.7370138

Orjuela, J.A. y M.M. Herrera. 2014. Perspective of traceability in the food supply chain: an approach from system dynamics. Ingeniería 19(2), 63-84. Doi: 10.14483/udistrital.jour.reving.2014.2.a03

Orjuela-Castro, J.A., A.L. Caicedo-Otavo, A.F. Ruiz-Moreno y W. Adarme-Jaimes. 2016a. Efecto de los mecanismos de integración externa en el desempeño logístico de cadenas Frutícolas. Un enfoque bajo dinámica de sistemas. Rev. Colomb. Cienc. Hortic. 10(2), 311-322. Doi: 10.17584/rcch.2016v10i2.5073

Orjuela, J.A., I. Castañeda, J. Canal y J. Rivera. 2015b. La logística en la cadena de frutas. Rev. Frutas y Hortalizas 39, 10-15

Orjuela-Castro, J.A., D.A. Sepulveda-Garcia e I.D. Ospina-Contreras. 2016b. Effects of using multimodal transport over the logistics performance of the food chain of uchuva. In: Figueroa-García, J., E. López-Santana y R. Ferro-Escobar (eds.). Applied Computer Sciences in Engineering. WEA 2016. Communications in Computer and Information Science 657 Doi: 10.1007/978-3-319-50880-1_15

Orjuela-Castro, J.A., N. Suárez-Camelo e Y.I. Chinchilla-Ospina (2016c). Costos logísticos y metodologías para el costeo en cadenas de suministro: una revisión de la literatura. Cuad. Contab., 17(44), 377-420. Doi: 10.11144/Javeriana.cc17-44.clmc

Quintero, V., G. Giraldo, J. Lucas y J. Vasco. 2013. Caracterización fisicoquímica del mango común (Mangifera indica L.) durante su proceso de maduración. Biotecnol. Sector Agropecu. Agroind. 11(1), 10-18.

Ruiz, A.F., A.L. Caicedo y J.A. Orjuela. 2015. External integration on agri-food supply chain: A review to the state of the art. Ingeniería 20(2), 9-30.

Sterman, J. 2000. Bussiness dynamics: Systems thinking. McGraw Hill, New York, NY, USA.

Taylor, D.H. 2005. Value chain analysis: an approach to supply chain improvement in agri-food chains. Int. J. Phys. Distrib. Logist. Manag. 35(10), 744-761. Doi: 10.1108/09600030510634599

Yasarcan, H. 2011. Information sharing in supply chains: A systemic approach pp. 4042-4060. En: 29th International Conference of the System Dynamics Society, Washington, DC. 